\title{
Transmission congestion tracing technique and its application to recognize weak parts of bulk power systems
}

\author{
Ming GAN ${ }^{1,2}$, Kaigui XIE ${ }^{1}$, Chunyan $\mathrm{LI}^{1}$
}

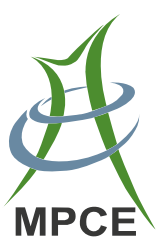

\begin{abstract}
A bulk power system is conventionally characterized by a complex structure with a large number of components. Each component generally has a different contribution to the transmission congestion (TC) of a system. Thus, a TC sharing method that can be used to evaluate the contribution of each component to the system TC and recognize the weak parts from the perspective of TC should be built. This paper presents a transmission congestion tracing (TCT) principle based on the failed component sharing principle and proportional sharing principle and a TCT model using the Monte Carlo simulation method. Case studies on the IEEE Reliability Test System indicate that the proposed method is effective and feasible.
\end{abstract}

Keywords Transmission congestion, Congestion indices, Congestion tracing principle, Component congestion contribution, Recognizing weak parts

CrossCheck date: 28 June 2016

Received: 16 October 2015/Accepted: 28 June 2016/Published online: 27 August 2016

(c) The Author(s) 2016. This article is published with open access at Springerlink.com

$\bowtie$ Kaigui XIE

kaiguixie@vip.163.com

Ming GAN

gm_225@163.com

Chunyan LI

lichunyancq@tom.com

1 State Key Laboratory of Power Transmission Equipment and System Security, Chongqing University, Chongqing, China

2 Department of Information Engineering, Logistical Engineering University, Chongqing, China

\section{Introduction}

A bulk power system (BPS) is generally characterized by a complex structure with a large number of components, such as generating units, transmission lines, and transformers. Each component exerts a different effect on the transmission congestion (TC) of a BPS. A functional TC sharing method can be used to quantify the contribution of each component to the TC of a BPS. Using this method, the key components that cause TC can be determined, and the weak parts of a BPS can be recognized. This method is also beneficial to optimize the type, number, and location of the flexible AC transmission system components, power system operations, and maintenance plans.

TC management is one of the major tasks performed by system operators to ensure that the operation of a transmission system is within operating limits [1]. Considerable work has been undertaken to develop TC models, TC elimination measures [2-5], TC benefit-cost analyses [6-11], and TC evaluation models and algorithms $[12,13]$.

Numerous studies have been conducted on power system reliability evaluation models and indices, such as the loss of load probability, loss of load expectation, and expected energy not supplied [14-17]. At present, the tracing technique has been applied to trace power flow [18-20], as well as in the unreliability of general complex networks, generation systems, and BPS [21-24]. The power tracing method allows the assessment of the contributions of individual generators (or loads) to individual line flows, which often utilize sensitivity analysis. This method can determine the relation between generator/load nodes and transmission lines in a power network and verify changes in the line flow along with the change in nodal generation/demand. Thus, the weak parts of a system can 
be recognized according to the change value [18-20]. For reliability tracing, the key components that cause unreliability are obtained according to reliability principles, that is, the failed component sharing principle (FCSP) and proportional sharing principle (PSP), and the reliability tracing results are the direct contribution of the weak parts to risks [21-24]. Both the sensitivity analysis and reliability tracing method can be used to recognize the key components that cause risk. Compared with sensitivity analysis, some of the advantages of the reliability tracing method are as follows.

1) Sensitivity analysis can test the effect of a component on risk in a system state. When several components fail simultaneously, the contribution of each failed component to risk cannot be estimated by sensitivity analysis. By contrast, the reliability tracing method can simultaneously analyze and estimate the contribution portion of multiple failed components to risk.

2) Sensitivity analysis repeatedly evaluates the contribution portion of every failed component to risk, whereas the reliability tracing method conducts only a one-time estimation to obtain the risk sharing indices and further improve the computational efficiency.

Considerable work has been conducted on the tracing techniques in power systems, including power flow tracing and unreliability tracing, and in the TC field, which is mainly focused on TC models, TC elimination measures, TC benefit-cost analysis, and TC evaluation. Unfortunately, no work has been conducted on the technique for tracing TC contributions and recognizing the weak parts of bulk power systems.

Considering the uncertainty of random failures of components, such as generating units, transmission lines, and transformers, a previous study [13] presented TC indices for the transmission components and overall system, which can be used to describe the TC degree. The present study utilizes a similar idea proposed in [21-24] to trace the TC indices suggested in [13]. Thus, this paper aims to recommend a recognition method for weak parts based on TC.

Notably, congestion under normal conditions frequently occurs because of heavy load growth without a good shedding load policy. In other words, a good shedding load policy can reduce and even eliminate the occurrence of congestion. Thus, only TC tracing (TCT) problems caused by failed components are discussed in this paper.

The paper proposes FCSP and PSP and establishes a TCT model and algorithm using the Monte Carlo technique. According to the TCT results, the weak system parts that cause TC can be recognized.

\section{TC indices of power systems}

The TC indices proposed in [13] are as follows. Among these indices, the first four are the indices for transmission components, and the rest are for power systems.

1) In a given time period, the occurrence probability of $\mathrm{TC}$ at transmission component $i$ is

$T L C P_{i}=\sum_{t \in D} P_{t}$

where $D$ is the system state set of TC occurring at the $i^{\text {th }}$ transmission component; $P_{t}$ is the probability of the $t^{\text {th }}$ system state.

2) In a given time period, the TC frequency occurring at transmission component $i$ is

$T L C F_{i}=\sum_{t \in D} F_{t}=\sum_{t \in D} P_{t} \sum_{k=1}^{N} \lambda_{k}$

where $F_{t}$ is the occurrence frequency of the $t^{\text {th }}$ system state; $N$ is the number of components in a power system; $\lambda_{k}$ is the transition rate of the $k^{\text {th }}$ component in state $D$. If the $k^{\text {th }}$ component is up, then $\lambda_{k}$ is the failure rate; otherwise, $\lambda_{k}$ is the repair rate.

3) In a given time period, the maximum congestion capacity of transmission component $i$ is

$T L C C_{i-\max }=\max _{t \in D}\left(T L C C_{i t}\right)$

where $T L C C_{i t}$ is the congestion capacity of transmission component $i$ at the $t^{\text {th }}$ state and $T L C C_{i t}=C_{i t}-C_{\text {rated }} ; C_{i t}$ and $C_{\text {rated }}$ are the actual capacity at the $t^{\text {th }}$ state and the rated capacity of the $i^{\text {th }}$ transmission component, respectively.

4) In a given time period (one year), the congestion energy of the $i^{\text {th }}$ transmission component is

$T L C E_{i}=\sum_{t \in D} T L C C_{i t} \times T L C P_{i t} \times 8760$

where $T L C P_{i t}$ is the probability of transmission component $i$ at the $t^{\text {th }}$ system state.

5) In a given time period, the occurrence probability of $\mathrm{TC}$ in a power system is

$S C P=\sum_{t \in S} P_{t}$

where $S$ is the set of all the TC system states.

6) The system congestion frequency is given by

$S C F=\sum_{t \in S} F_{t}=\sum_{t \in S} P_{t} \sum_{k=1}^{N} \lambda_{k}$

7) The maximum system congestion capacity is 
$S C C_{\max }=\max _{t \in S}\left(\sum_{i=1}^{l} T L C C_{i t}\right)$

where $l$ is the number of congested transmission components at the $t^{\text {th }}$ system state.

8) The system congestion energy is given by

$S C E=\sum_{t \in S} P_{t} \times S C C_{t} \times 8760$

where $S C C_{t}$ is the system congestion capacity at the $t^{\text {th }}$ system state.

\section{TCT principle}

The above-cited study [13] proposed TC indices by considering the random failures of power components using the Monte Carlo technique. Assuming that a simulated system state occurs with a given SCP because of the simultaneous failure of three components, the questions that arise are as follows: How much is the "contribution" of a particular component to the SCP? What are the major TC contributions in a power system?

Two basic TCT principles are proposed for TCT in BPS.

\section{1) FCSP}

The obligation for a TC system state is assigned to the failed components. In other words, healthy components have no obligation for a TC system state. TC does not generally occur in a normal state. Thus, a TC index should be shared by the failed components for a TC system state.

2) PSP

The TC indices are proportionally shared among failed components.

According to the TCT principles described above, a proportional sharing method suitable for the Monte Carlo technique can be obtained.

Certain failed components notably exert no effect on a power system TC; thus, they do not share in the TC responsibility. Failed transmission components should generally share the TC responsibility directly because of their effect on TC, and these effective failed components contribute to TC. However, certain generating units possibly exert no effect on TC. Thus, the generating units that are responsible for $\mathrm{TC}$ should be filtered from the failed generating units. A combination of the Monte Carlo and enumeration methods is used to filter the failed generating units in this paper. The algorithm can be summarized in the following steps.

Step 1 Assume that $v$ components in a system fail simultaneously, and TC occurs ( $e$ is a system congestion index A). " $U$ " failed generating units among all the failed components are denoted as $q_{1}, q_{2}, \ldots, q_{u}$.

Step 2 Let the $i^{\text {th }}$ failed generating unit $q_{i}$ recover a healthy component from a failed component, and calculate the TC index A as $f$.

Step 3 If $f=e$, then the generating unit $q_{i}$ exerts no effect on TC, so that the generating unit is a noneffective failed component that does not contribute to TC. If $f<e$, then the generating unit exerts an effect on TC, so that the generating unit is an effective failed component that contributes to TC.

Step 4 Repeat Steps 2 and 3 until all the failed generating units are enumerated. The effective failed generating units, which should share TC responsibility, are filtered in this manner.

Evidently, the failed components for FCSP and PSP are effective failed components.

We assume that $t$ is a TC system state simulated by the Monte Carlo technique because of the simultaneous failure of $m$ effective failed components. Let $\varphi_{t}$ be a TC index of system state $t$.

In a system state simulated by the Monte Carlo technique, each effective failed component possesses an equal obligation for a failure system state based on PSP. Thus, each effective failed component $j$ among $m$ effective failed components should be assigned $1 / m$ contribution to the TC index $\varphi_{t}$, which is given by

$\varphi(t \rightarrow j)=\frac{1}{m} \varphi_{t} \quad j=1,2, \cdots, m$

As shown in (1), PSP possesses an identity. In other words, TC indices can be shared equally and completely.

\section{Tracing TC indices for BPS}

We assume that a BPS has $N$ components and each component presents only two states: up and down. Using the TCT principle, TCT models can be obtained for the transmission components and the system.

\subsection{Tracing models for TC indices of transmission component}

1) Tracing model for TC index $T L C P_{i}$

According to the PSP described above, the $T L C P_{i}$ index tracing model for the effective failed component $j$ is given by

$T L C P_{i}(D \rightarrow j)=\sum_{t \in D} \frac{P_{t}}{m_{t}}$ 
where $m_{t}$ is the number of effective failed components in the $t^{\text {th }}$ TC state $t$.

2) Tracing model for index $T L C F_{i}$

The $T L C F_{i}$ index sharing model for the effective failed component $j$ is given by

$T L C F_{i}(D \rightarrow j)=\sum_{t \in D} \frac{F_{t}}{m_{t}}$

3) Tracing model for indices $T L C C_{i-\max }$ and $T L C E_{i}$

Similarly, the $T L C C_{i-\max }$ and $T L C E_{i}$ sharing models for the effective failed component $j$ are given by

$$
\begin{aligned}
& T L C C_{i-\max }(D \rightarrow j)=\frac{T L C C_{i-\max }}{m_{t}} \\
& T L C E_{i}(D \rightarrow j)=\sum_{t \in D} \frac{P_{t}}{m_{t}} \times T L C C_{i t} \times 8760
\end{aligned}
$$

\subsection{Tracing models for system TC indices}

1) Tracing model for index $S C P$

The $S C P$ tracing model for the effective failed component $j$ is given by

$S C P(S \rightarrow j)=\sum_{t \in S} \frac{P_{t}}{m_{t}}$

2) Tracing model for index $S C F$

The $S C F$ tracing model for the effective failed component $j$ is given by

$S C F(S \rightarrow j)=\sum_{t \in S} \frac{F_{t}}{m_{t}}$

3) Tracing model for index $S C C_{\max }$ and $S C E$

Similarly, the $S C C_{\max }$ and $S C E$ tracing models for the effective failed component $j$ are given by

$S C C_{\max }(S \rightarrow j)=\frac{S C C_{\max }}{m_{t}}$

$S C E(S \rightarrow j)=\sum_{t \in S} \frac{P_{t}}{m_{t}} \times S C C_{t} \times 8760$

The TCT sharing factor (TCTSF) can be used to describe the degree of TC contribution per unit.

The TCTSF based on the $T L C P_{i}$ for the effective failed component $j$ is given by $\operatorname{TCTSF}_{P i}=\operatorname{TLCP}_{i}(D \rightarrow j) / T L C P_{i}$. The other indices can also be defined in the same manner.

For a given component, a greater magnitude of the TCTSF translates to a greater significance of the TC effect of the component on the system. The components with large TCTSF are the weak system parts.

Table 1 provides the TCTSF indices and calculation models of the effective failed component $j$.
Table 1 TCTSF indices and models of effective failed component $j$

\begin{tabular}{ll}
\hline Denotation & Calculation formula \\
\hline$T_{C T S F_{P i}}$ & $T L C P_{i}(D \rightarrow j) / T L C P_{i}$ \\
$\operatorname{TCTSF}_{F i}$ & $T L C F_{i}(D \rightarrow j) / T L C F_{i}$ \\
$\operatorname{TCTSF}_{C i \text {-max }}$ & $T L C C_{i-\max }(D \rightarrow j) / T L C C_{i-\max }$ \\
$\operatorname{TCTSF}_{E i}$ & $T L C E_{i}(D \rightarrow j) / T L C E_{i}$ \\
$\operatorname{TCTSF}_{P}$ & $S C P(S \rightarrow j) / S C P$ \\
$\operatorname{TCTSF}_{F}$ & $S C F(S \rightarrow j) / S C F$ \\
$\operatorname{TCTSF}_{C \max }$ & $S C C_{\text {max }}(S \rightarrow j) / S C C_{\text {max }}$ \\
$\operatorname{TCTSF}_{E}$ & $S C E(S \rightarrow j) / S C E$ \\
\hline
\end{tabular}

\section{TC tracing algorithm for BPS}

A TCT algorithm for BPS can be summarized as follows.

Step 1 Calculate the TC indices for the transmission components $T L C P_{i}, \quad T L C F_{i}, \quad T L C C_{i-\max }$ and $T L C E_{i}$, as well as the system TC indices $S C P$, $S C F, S C C_{\max }$ and SCE [13].

Step 2 Calculate the contribution of the effective failed component $j$ to the transmission component and system indices using (2) to (5) and (6) to (9), respectively.

Step 3 Repeat Step 2 and calculate the contribution of each TC index of the other effective failed components.

Step 4 According to the indices and models in Table 1, calculate the TCTSF indices and identify the weak parts of a BPS.

\section{Case studies}

Case studies on the IEEE reliability test system (IEEERTS) are used to verify the effectiveness of the proposed model. Figure 1 shows a single line diagram of IEEE-RTS. The system includes 24 buses, $33 \mathrm{AC}$ transmission lines, 5 transformers, and 32 generating units. The total generation capacity is 3405 MW and the peak load is $2850 \mathrm{MW}$. The relevant electrical and reliability parameters of the components are shown in [25]. A constant load, that is, the peak load, and load curtailment philosophy, such as Pass-I Policy and Average Policy [13], are used in the proposed TCT models. The non-sequential Monte Carlo technique with 1 million simulation replications is used to analyze the system. After a random sampling of the state of the generating units, transmission lines, and transformers, the power deviation between the total generation and load demands can be calculated. If the power deviation is negative, then the load is shed. Otherwise, the generating units 
are re-dispatched, and the system power flow is calculated. The results of the power flow are used to identify whether or not the transmission components are congested.

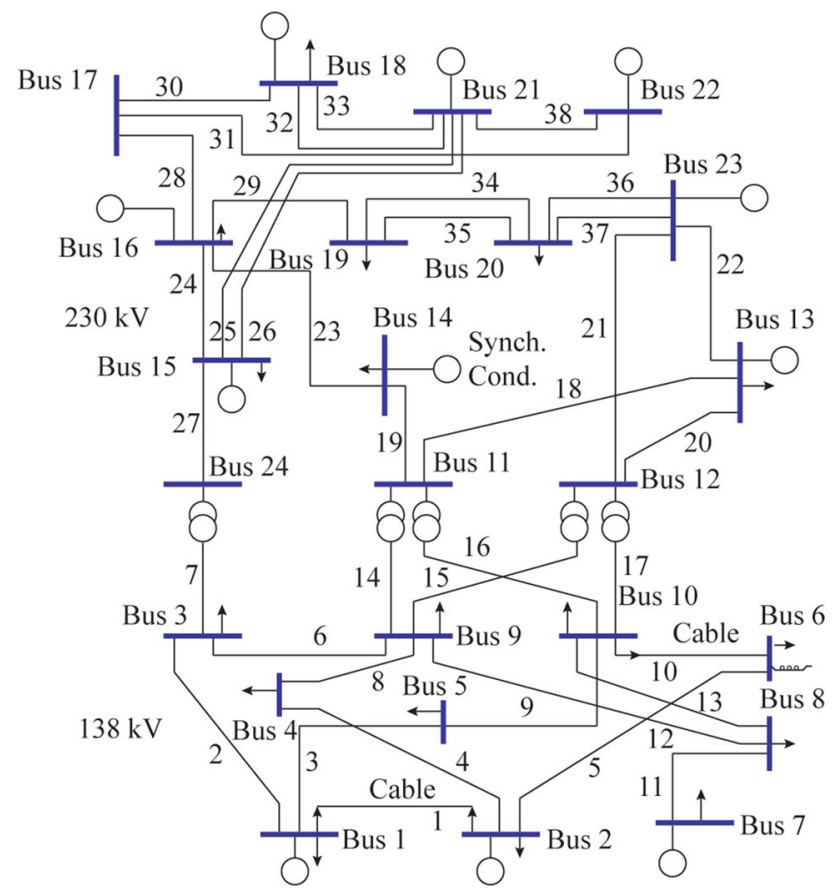

Fig. 1 Single line diagram of IEEE-RTS
The simulation program was coded using MATLAB 7.3 based on the proposed techniques and was run on a computer with a $2.4 \mathrm{GHz}$ Intel Core i5-6200U CPU and 4 GB RAM. The computation time of 1 million simulations of the base case (Pass-I Policy) is 4.8 hours.

\subsection{TCT and recognizing weak parts on Pass-I Policy}

Based on [13], a constant load or peak load and Pass-I Policy are used in this analysis. Eight transmission components are congested because of the random failure of components. Table 2 shows the TC indices for these transmission components and system. L6 presents the most severe TC which has the largest TC indices.

\subsubsection{TCT for transmission components and system}

Tables 3 and 4 show the TCT and TCTSF indices associated with the TC of L6 and the system, respectively.

\subsubsection{TCT analysis of transmission components and system}

Tables 3 and 4 show that the sum of every TCTSF index for the components is $100 \%$, including TCTSF $_{P i}, T C T S F_{F i}$ and $T C T S F_{E i}$ for the component, TCTSF $P, T C T S F_{F}$,

Table 2 TC indices for transmission components and system (constant load model, Pass-I Policy)

\begin{tabular}{lllll}
\hline Transmission components & $T L C P_{i}$ & $T L C F_{i}$ (times/year) & $T L C C_{i-\text { max }}(\mathrm{MVA})$ & $T L C E_{i}(\mathrm{MWh} / \mathrm{year})$ \\
\hline L6 & $4.0 \times 10^{-6}$ & 0.0081 & 168.17 & 2.24 \\
L7 & $2.0 \times 10^{-6}$ & 0.0037 & 210.00 & 1.98 \\
L13 & $3.0 \times 10^{-6}$ & 0.0048 & 12.92 & 0.34 \\
L24 & $1.0 \times 10^{-6}$ & 0.0011 & 42.95 & 0.38 \\
L25 & $1.0 \times 10^{-6}$ & 0.0025 & 162.67 & 1.43 \\
L27 & $1.0 \times 10^{-6}$ & 0.0019 & 120.00 & 1.05 \\
L28 & $1.0 \times 0^{-6}$ & 0.0011 & 57.59 & 0.50 \\
L29 & $1.0 \times 10^{-6}$ & 0.0014 & 92.12 & 0.81 \\
\hline
\end{tabular}

Note: System indices $S C P=1.1 \times 10^{-5}, S C F=0.0189$ times/year, $S C C_{\max }=498.17 \mathrm{MVA}, S C E=8.72 \mathrm{MWh} /$ year

Table 3 TCT and TCTSF indices associated with TC of L6 (constant load model, Pass-I Policy)

\begin{tabular}{|c|c|c|c|c|c|c|}
\hline \multirow[t]{2}{*}{ Component } & \multicolumn{3}{|c|}{ TCT indices for L6 } & \multicolumn{3}{|l|}{ TCTSF } \\
\hline & $T L C P_{i}$ & $T L C F_{i}$ (times/year) & $T L C E_{i}(\mathrm{MWh} /$ year $)$ & $\operatorname{TCTSF}_{P i}(\%)$ & $\operatorname{TCTSF}_{F i}(\%)$ & $\operatorname{TCTSF}_{E i}(\%)$ \\
\hline L2 & $1.00 \times 10^{-6}$ & 0.00165 & 0.225 & 25.00 & 20.40 & 10.06 \\
\hline L19 & $5.00 \times 10^{-7}$ & 0.00120 & 0.159 & 12.50 & 14.80 & 7.08 \\
\hline L23 & $5.00 \times 10^{-7}$ & 0.00120 & 0.737 & 12.50 & 14.80 & 32.86 \\
\hline L27 & $1.00 \times 10^{-6}$ & 0.00165 & 0.225 & 25.00 & 20.40 & 10.06 \\
\hline L29 & $1.00 \times 10^{-6}$ & 0.00240 & 0.895 & 25.00 & 29.60 & 39.94 \\
\hline All transmission components & & & & 100 & 100 & 100 \\
\hline All generating units & & & & 0 & 0 & 0 \\
\hline Total & $4 \times 10^{-6}$ & 0.0081 & 2.24 & 100 & 100 & 100 \\
\hline
\end{tabular}


TCTSF $_{\text {Cmax }}$ and $T C T S F_{E}$ for the system. In other words, the TC indices for a BPS can be completely distributed among all the components using the proposed TCT technique.

Table 3 shows that the TC of L6 is caused by the failures of the transmission components based on 1 million simulation samplings and Pass-I Policy, which all account for $100 \%$ of the indices TCTSF $_{P i}$, TCTSF $_{F i}$ and TCTSF $E i$. Among the transmission components, L23 and L29 exert the greatest effect on the TC of L6, which accounts for $32.86 \%$ and $39.94 \%$ of the index TCTSF $E$, respectively. The two transmission components account for $37.50 \%$, $44.40 \%$ and $72.80 \%$ of TCTSF $_{P i}$, TCTSF Fi $_{F i}$ and TCTSF $E$, respectively. Thus, these two components are the weak parts that cause the TC of L6, which is due to the occurrence of TC at L6 when L23 and L29 are simultaneously in a down state. Under this state, L6 presents the greatest congestion capacity among all its TC states.

Table 4 shows that the transmission components L2, L7, L12, L23, L27, L28 and L29 exert a relatively large effect on the indices $S C P$ and $S C F$. These seven components account for $68.18 \%$ and $67.51 \%$ of the indices TCTSF $_{P}$ and $\operatorname{TCTSF}_{F}$. However, L2, L12 and L27 possess relatively larger TCTSF $_{P}$ and $T C T S F_{F}$ and a smaller $T C T S F_{E}$, which is due to the fact that the TC of these transmission components only occurs in the case of the failure of two and more component, and presents a smaller congestion capacity during each TC state.

Figure 2 shows the TCTSF indices of the system TCT based on Pass-I Policy. Figure 1 shows that L23 and L29 have a significant effect on the index $S C E$, which accounts

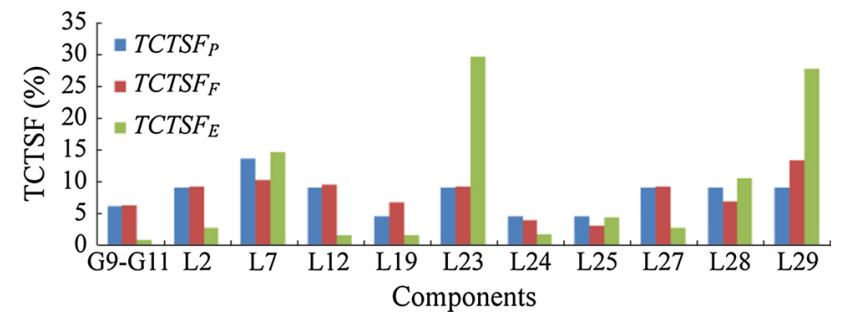

Fig. 2 System TCTSF (constant load model, Pass-I Policy)

for $57.68 \%$ of the index $T C T S F_{E}$. For the indices $S C P$, $S C F$, and $S C E$, the following seven components are the weak parts from the perspective of TC: L2, L7, L12, L23, L27, L28 and L29.

Figure 2 shows that the major components that cause TC are the transmission components when using Pass-I Policy.

\subsection{TCT and recognizing weak parts on Average Policy}

Based on [13], the system TC occurs at eight transmission components because of the random failure of components while using Average Policy. Table 5 shows the TC indices for the eight congested transmission components and system. L11 shows the most severe TC because its TC indices are the largest.

Table 5 shows that the other seven congested transmission components exhibit a lower occurrence probability of TC than L11, whereas their TC capacity is relatively

Table 4 TCT and TCTSF indices associated with TC of system (constant load model, Pass-I Policy)

\begin{tabular}{|c|c|c|c|c|c|c|c|c|c|}
\hline \multirow[t]{2}{*}{ Component } & \multirow{2}{*}{$\begin{array}{l}\text { Capacity } \\
\text { (MW)/Bus }\end{array}$} & \multicolumn{4}{|c|}{ System TCT indices } & \multicolumn{4}{|l|}{ TCTSF } \\
\hline & & $S C P$ & $\begin{array}{l}S C F \\
\text { (times/year) }\end{array}$ & $\begin{array}{l}S C C_{\max } \\
\text { (MVA) }\end{array}$ & $\begin{array}{l}S C E \\
\text { (MWh/year) }\end{array}$ & $\begin{array}{l}\operatorname{TCTSF}_{P} \\
(\%)\end{array}$ & $\begin{array}{l}\operatorname{TCTSF}_{F} \\
(\%)\end{array}$ & $\begin{array}{l}\text { TCTSF } F_{\text {max }} \\
(\%)\end{array}$ & $\begin{array}{l}\operatorname{TCTSF}_{E} \\
(\%)\end{array}$ \\
\hline G9, G10, G11 & 100/Bus7 & $6.67 \times 10^{-7}$ & 0.00120 & 0 & 0.075 & 6.06 & 6.33 & 0 & 0.86 \\
\hline All generating units & & & & & & 18.17 & 18.97 & 0 & 2.61 \\
\hline L2 & & $1.00 \times 10^{-6}$ & 0.00174 & 0 & 0.243 & 9.09 & 9.18 & 0 & 2.78 \\
\hline L7 & & $1.50 \times 10^{-6}$ & 0.00194 & 0 & 1.283 & 13.64 & 10.24 & 0 & 14.71 \\
\hline $\mathrm{L} 12$ & & $1.00 \times 10^{-6}$ & 0.00180 & 0 & 0.129 & 9.09 & 9.49 & 0 & 1.48 \\
\hline L19 & & $5.00 \times 10^{-7}$ & 0.00126 & 0 & 0.132 & 4.55 & 6.66 & 0 & 1.51 \\
\hline L23 & & $1.00 \times 10^{-6}$ & 0.00174 & 249.09 & 2.603 & 9.09 & 9.23 & 50 & 29.84 \\
\hline L24 & & $5.00 \times 10^{-7}$ & 0.00073 & 0 & 0.143 & 4.55 & 3.84 & 0 & 1.64 \\
\hline L25 & & $5.00 \times 10^{-7}$ & 0.00057 & 0 & 0.374 & 4.55 & 3.02 & 0 & 4.28 \\
\hline L27 & & $1.00 \times 10^{-6}$ & 0.00174 & 0 & 0.243 & 9.09 & 9.18 & 0 & 2.78 \\
\hline L28 & & $1.00 \times 10^{-6}$ & 0.00130 & 0 & 0.918 & 9.09 & 6.86 & 0 & 10.53 \\
\hline L29 & & $1.00 \times 10^{-6}$ & 0.00252 & 249.09 & 2.428 & 9.09 & 13.33 & 50 & 27.84 \\
\hline $\begin{array}{l}\text { All transmission } \\
\text { components }\end{array}$ & & & & & & 81.83 & 81.03 & 100 & 97.39 \\
\hline Total & & $1.1 \times 10^{-5}$ & 0.0189 & 498.17 & 8.72 & 100 & 100 & 100 & 100 \\
\hline
\end{tabular}


Table 5 TC indices for transmission components (constant load model, Average Policy)

\begin{tabular}{llll}
\hline Transmission components & $T L C P_{i}$ & $T L C F_{i}$ (times/year) & $T L C E_{i}(\mathrm{MWh} / \mathrm{year})$ \\
\hline L11 & $2.6 \times 10^{-4}$ & 0.2308 & 7.17 \\
L6 & $4.0 \times 10^{-6}$ & 0.0081 & 2.24 \\
L7 & $2.0 \times 10^{-6}$ & 0.0037 & 1.98 \\
L13 & $3.0 \times 10^{-6}$ & 0.0048 & 0.34 \\
L25 & $1.0 \times 10^{-6}$ & 0.0025 & 1.43 \\
L27 & $1.0 \times 10^{-6}$ & 0.0019 & 1.05 \\
L28 & $1.0 \times 10^{-6}$ & 0.0011 & 0.50 \\
L29 & $1.0 \times 10^{-6}$ & 0.0014 & 0.81 \\
\hline
\end{tabular}

Note: System indices $S C P=2.72 \times 10^{-4}, S C F=0.2500$ times/year, $S C E=15.56 \mathrm{MWh} /$ year

Table 6 TCT and TCTSF indices associated with TC of L11 (constant load model, Average Policy)

\begin{tabular}{|c|c|c|c|c|c|c|c|}
\hline \multirow[t]{2}{*}{ Component } & \multirow[t]{2}{*}{ Capacity (MW)/Bus } & \multicolumn{3}{|c|}{ TCT indices for L11 } & \multicolumn{3}{|l|}{ TCTSF } \\
\hline & & $T L C P_{i}$ & $\begin{array}{l}T L C F_{i} \\
\text { (times/year) }\end{array}$ & $\begin{array}{l}T L C E_{i} \\
(\mathrm{MWh} / \text { year) }\end{array}$ & $\begin{array}{l}\operatorname{TCTSF}_{P i} \\
(\%)\end{array}$ & $\begin{array}{l}\operatorname{TCTSF}_{F i} \\
(\%)\end{array}$ & $\begin{array}{l}\operatorname{TCTSF}_{E i} \\
(\%)\end{array}$ \\
\hline G9, G10, G11 & 100/Bus7 & $1.81 \times 10^{-5}$ & 0.01595 & 0.586 & 6.95 & 6.9 & 8.18 \\
\hline G12, G13, G14 & 197/Bus13 & $7.22 \times 10^{-6}$ & 0.00721 & 0.172 & 2.78 & 3.1 & 2.39 \\
\hline G20 & 155/Bus 15 & $6.31 \times 10^{-6}$ & 0.00652 & 0.213 & 2.43 & 2.8 & 2.97 \\
\hline G21 & 155/Bus16 & $5.87 \times 10^{-5}$ & 0.05051 & 1.520 & 22.57 & 21.89 & 21.21 \\
\hline G22 & 400/Bus18 & $5.91 \times 10^{-5}$ & 0.05075 & 1.520 & 22.73 & 21.99 & 21.20 \\
\hline G23 & 400/Bus21 & $8.36 \times 10^{-6}$ & 0.00858 & 0.240 & 3.21 & 3.72 & 3.34 \\
\hline G30, G31 & 155/Bus23 & $5.56 \times 10^{-5}$ & 0.04730 & 1.456 & 21.38 & 20.50 & 20.31 \\
\hline G32 & 350/Bus23 & $1.81 \times 10^{-5}$ & 0.01595 & 0.586 & 6.95 & 6.91 & 8.18 \\
\hline All generating units & & & & & 99.17 & 98.48 & 99.30 \\
\hline L28 & - & $9.75 \times 10^{-7}$ & 0.00182 & 0.034 & 0.38 & 0.79 & 0.48 \\
\hline L33 & - & $1.95 \times 10^{-7}$ & 0.00031 & 0.001 & 0.08 & 0.14 & 0.02 \\
\hline L35 & - & $9.75 \times 10^{-7}$ & 0.00137 & 0.015 & 0.38 & 0.60 & 0.21 \\
\hline $\begin{array}{l}\text { All transmission } \\
\text { components }\end{array}$ & & & & & 0.83 & 1.52 & 0.70 \\
\hline Total & & $2.60 \times 10^{-4}$ & 0.23080 & 7.170 & 100 & 100 & 100 \\
\hline
\end{tabular}

large. Thus, the transmission components exert a comparatively large effect on $S C E$.

\subsubsection{TCT for transmission components and system}

Tables 6 and 7 show the TCT and TCTSF indices associated with the TC of L11 and the system, respectively. The TC indices for a BPS can also be completely distributed among all the components.

\subsubsection{TCT analysis of transmission components and system}

Figure 3 shows the TCTSF indices of the system TCT using Average Policy, with G22, G23 and G32 exerting the greatest effect on the indices $S C P$ and $S C F$. These three components account for $64.03 \%$ and $59.52 \%$ of indices TCTSF $_{P}$ and TCTSF $_{F}$. A similar conclusion can be drawn using Pass-I Policy. The transmission components L23 and L29 have a significant effect on the index SCE, which accounts for $30.97 \%$ of index TCTSF $_{E}$. For Pass-I Policy, when the BPS encounters a severe contingency that requires load curtailment, the loads are curtailed at the buses that are closest to the elements on outage. For Average Policy, when the BPS encounters a severe contingency that requires load curtailment, all the loads at the buses are curtailed with a similar proportion. For IEEERTS, G22, G23 and G32 present the largest or second largest equivalent unavailable capacity (generation capacity $\times$ unavailability) among all the generating units. If the total capacity of the generating units is less than the load 
Table 7 TCT and TCTSF indices associated with TC of system (constant load model, Average Policy)

\begin{tabular}{|c|c|c|c|c|c|c|c|c|c|}
\hline \multirow[t]{2}{*}{ Component } & \multirow{2}{*}{$\begin{array}{l}\text { Capacity } \\
\text { (MW)/Bus }\end{array}$} & \multicolumn{4}{|c|}{ System TCT indices } & \multicolumn{4}{|l|}{ TCTSF } \\
\hline & & $S C P$ & $\begin{array}{l}S C F \\
\text { (times/year) }\end{array}$ & $\begin{array}{l}S C C_{\max } \\
\text { (MVA) }\end{array}$ & $\begin{array}{l}S C E \\
\text { (MWh/year) }\end{array}$ & $\begin{array}{l}\operatorname{TCTSF}_{P} \\
(\%)\end{array}$ & $\begin{array}{l}\operatorname{TCTSF}_{F} \\
(\%)\end{array}$ & $\begin{array}{l}\text { TCTSF }_{C \max } \\
(\%)\end{array}$ & $\begin{array}{l}\operatorname{TCTSF}_{E} \\
(\%)\end{array}$ \\
\hline G9 G10 G11 & 100/Bus7 & $6.55 \times 10^{-7}$ & 0.00109 & 0 & 0.072 & 0.24 & 0.44 & 0 & 0.46 \\
\hline G12 G13 G14 & 197/Bus13 & $1.82 \times 10^{-5}$ & 0.01597 & 0 & 0.585 & 6.68 & 6.39 & 0 & 3.76 \\
\hline G20 & 155/Bus15 & $7.25 \times 10^{-6}$ & 0.00722 & 0 & 0.171 & 2.67 & 2.89 & 0 & 1.10 \\
\hline G21 & 155/Bus16 & $6.34 \times 10^{-6}$ & 0.00653 & 0 & 0.213 & 2.33 & 2.61 & 0 & 1.37 \\
\hline $\mathrm{G} 22$ & 400/Bus18 & $5.90 \times 10^{-5}$ & 0.05060 & 0 & 1.516 & 21.68 & 20.24 & 0 & 9.74 \\
\hline G23 & 400/Bus21 & $5.94 \times 10^{-5}$ & 0.05084 & 0 & 1.516 & 21.82 & 20.33 & 0 & 9.74 \\
\hline G30 G31 & 155/Bus23 & $8.40 \times 10^{-6}$ & 0.00860 & 0 & 0.239 & 3.09 & 3.44 & 0 & 1.54 \\
\hline G32 & 350/Bus23 & $5.58 \times 10^{-5}$ & 0.04739 & 0 & 1.452 & 20.53 & 18.95 & 0 & 9.33 \\
\hline All generating units & & & & & & 95.95 & 92.38 & 0 & 47.01 \\
\hline L2 & & $9.83 \times 10^{-7}$ & 0.00215 & 0 & 0.217 & 0.36 & 0.86 & 0 & 1.40 \\
\hline L7 & & $1.48 \times 10^{-7}$ & 0.00175 & 0 & 0.814 & 0.54 & 0.70 & 0 & 5.23 \\
\hline L12 & & $9.83 \times 10^{-7}$ & 0.00163 & 0 & 0.108 & 0.36 & 0.65 & 0 & 0.69 \\
\hline L19 & & $4.92 \times 10^{-7}$ & 0.00091 & 0 & 0.221 & 0.18 & 0.36 & 0 & 1.42 \\
\hline L23 & & $9.83 \times 10^{-7}$ & 0.00161 & 249.09 & 2.494 & 0.36 & 0.64 & 50 & 16.03 \\
\hline L24 & & $4.92 \times 10^{-7}$ & 0.00052 & 0 & 0.243 & 0.18 & 0.21 & 0 & 1.56 \\
\hline L25 & & $4.92 \times 10^{-7}$ & 0.00123 & 0 & 0.687 & 0.18 & 0.49 & 0 & 4.42 \\
\hline L27 & & $9.83 \times 10^{-7}$ & 0.00215 & 0 & 0.217 & 0.36 & 0.86 & 0 & 1.40 \\
\hline L28 & & $1.97 \times 10^{-7}$ & 0.00358 & 0 & 0.903 & 0.72 & 1.43 & 0 & 5.80 \\
\hline L29 & & $9.83 \times 10^{-7}$ & 0.00182 & 249.09 & 2.325 & 0.36 & 0.73 & 50 & 14.94 \\
\hline L33 & & $1.97 \times 10^{-7}$ & 0.00031 & 0 & 0.001 & 0.07 & 0.13 & 0 & 0.01 \\
\hline L35 & & $9.83 \times 10^{-7}$ & 0.00138 & 0 & 0.015 & 0.36 & 0.55 & 0 & 0.09 \\
\hline All transmission components & & & & & & 4.05 & 7.62 & 0 & 52.99 \\
\hline Total & & $2.72 \times 10^{-4}$ & 0.2500 & 498.17 & 15.56 & 100 & 100 & 100 & 100 \\
\hline
\end{tabular}

Note: To highlight the key components, components with a TC contribution of less than $2 \%$ are not shown in the table

demands, then the loads must be shed to keep the system in a normal state. Thus, these three components present a larger probability to cause load shedding in their down states than the other generating units. Based on Pass-I Policy, the loads at Bus 7 and Bus 8 should not be shed because of their far distance from the elements on outage. Based on Average Policy, the loads at Bus 7 and Bus 8 should be shed with the same proportion as that of the other loads used. Considering that the output of the generating units at Bus 7 remains unchanged, as well as its load curtailment, the power flow of L11 increases, which may lead to the occurrence of TC at L11. Thus, L11 shows the largest $T L C P_{i}$ and $T L C F_{i}$ among all the transmission components in the BPS, and its $T L C P_{i}$ or $T L C F_{i}$ is larger than the sum of the other seven transmission components. Evidently, SCP and SCF mainly depend on the $T L C P_{i}$ and $T L C F_{i}$ of $\mathrm{L} 11$.

Based on SCP, SCF and SCE, the weak parts that cause TC are G22, G23, G32, L23 and L29.
Figure 3 shows that the major components that cause TC are the generating units, as well as L23 and L29, using Average Policy. The failures of the generating unit have less effect on TC when using Pass-I Policy load curtailment philosophy than when using Average Policy.

The proposed TCT models can be used to distribute impartially and reasonably the TC indices to each effective failed component. According to the TCT results, the weak parts that cause TC can be recognized.

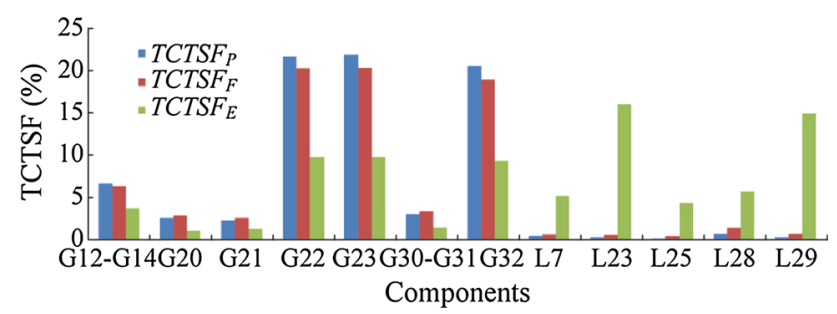

Fig. 3 System TCTSF (constant load model, Average Policy) 


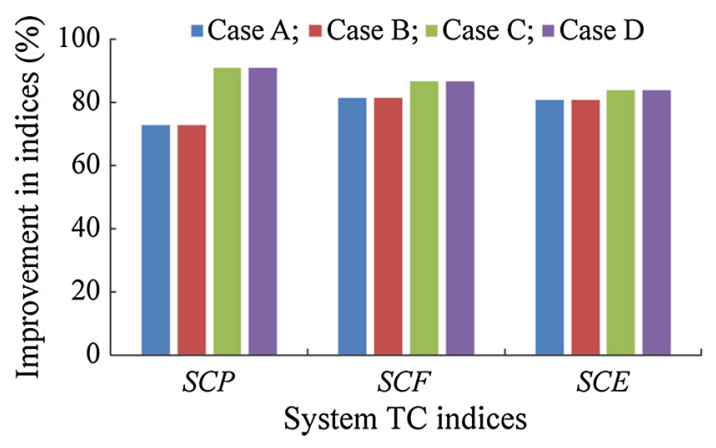

Fig. 4 Comparison of system TC indices of Cases A, B, C and D

\subsection{Comparison of improvement measures}

A constant load and Pass-I load curtailment policy are used in this section. Based on the analysis in Section 6.1, seven components are the weak parts from the perspective of TC. The transmission component L7 has the largest $\operatorname{TCTSF}_{P}$ and a relatively larger $T C T S F_{F}$ and $T C T S F_{E}$ than the other components, whereas the transmission component L28 has the smallest $\operatorname{TCTSF}_{P}$. Thus, four cases are analyzed.

Case A the failure rate of $\mathrm{L} 7$ is reduced by $50 \%$.

Case B the repair time of L7 is decreased by $50 \%$.

Case $\mathrm{C}$ the failure rate of $\mathrm{L} 28$ is lessened by $50 \%$.

Case D the repair time of L28 is diminished by $50 \%$.

Figure 4 shows the $S C P, S C F$ and $S C E$ indices for the BPS when the reliability performance of L7 and L28 are changed. The system $S C P$ is $9 \times 10^{-6}$ in Case A, which represents a drop of $9.09 \%$ compared with the $S C P$ in Case $\mathrm{C}$ and a drop of $18.19 \%$ compared with the $S C P$ in the base case. Similar conclusions can be drawn for the $S C F$ and $S C E$ indices of Cases $\mathrm{A}$ and $\mathrm{C}$ and for the indices of Cases $\mathrm{B}$ and D. These results indicate that, compared with L28, L7 is a weak part from the perspective of TC.

\section{Conclusion}

This paper presents TCT principles-FCSP and PSP, and builds TCT models using a similar idea employed in reliability tracing.

TCT models can be used to distribute the contribution of each component to TC indices and obtain the TCT indices of the transmission components and system, which can be used to recognize the weak parts of a power system that cause TC. The correctness and efficiency of the proposed TCT models are verified by case studies on IEEE-RTS. The proposed techniques are actually general methods for tracing TC without any special assumptions or requirements associated with power systems and can be extended to trace the TC of power systems containing wind power.

Acknowledgment This work was supported by National Natural Science Foundation of China (No. 51247006).

Open Access This article is distributed under the terms of the Creative Commons Attribution 4.0 International License (http:// creativecommons.org/licenses/by/4.0/), which permits unrestricted use, distribution, and reproduction in any medium, provided you give appropriate credit to the original author(s) and the source, provide a link to the Creative Commons license, and indicate if changes were made.

\section{References}

[1] Kumar A, Srivastava SC, Singh SN (2005) Congestion management in competitive power market: a bibliographical survey. Electr Power Syst Res 76(1/2/3):153-164

[2] Li CB, Xiao LW, Cao YJ et al (2014) Optimal allocation of multi-type FACTS devices in power systems based on power flow entropy. J Mod Power Syst Clean Energy 2(2):173-180. doi:10.1007/s40565-014-0059-x

[3] Dutta S, Singh SP (2008) Optimal rescheduling of generators for congestion management based on particle swarm optimization. IEEE Trans Power Syst 23(4):1560-1569

[4] Hosseini SA, Amjady N, Shafie-Khah M et al (2016) A new multi-objective solution approach to solve transmission congestion management problem of energy markets. Appl Energy 165:462-471

[5] Salehizadeh MR, Rahimi-Kian A, Oloomi-Buygi M (2015) Security-based multi-objective congestion management for emission reduction in power system. Int $\mathrm{J}$ Electr Power Energy Syst 65:124-135

[6] Liu K, Ni YX, Wu FF et al (2007) Decentralized congestion management for multilateral transactions based on optional resource allocation. IEEE Trans Power Syst 22(4):1835-1842

[7] Pandey SN, Tapaswi S, Srivastava L (2008) Nodal congestion price estimation in spot power market using artificial neural network. IEE P-Gener Transm Distrib 2(2):280-290

[8] Li G, Liu CC, Salazar H (2006) Forecasting transmission congestion using day-ahead shadow prices. In: Proceedings of the 2006 power systems conference and exposition (PSCE'06), Atlanta, 29 Oct-1 Nov 2006, pp 1705-1709

[9] Conejo AJ, Plazas MA, Espinola R et al (2005) Day-ahead electricity price forecasting using the wavelet transform and ARIMA models. IEEE Trans Power Syst 20(2):1035-1042

[10] Sarkar V, Khaparde SA (2008) A comprehensive assessment of the evaluation of financial transmission rights. IEEE Trans Power Syst 23(4):1783-1795

[11] Min L, Lee ST, Zhang P et al (2008) Short-term probabilistic transmission congestion forecasting. In: Proceedings of the 3th international conference on electric utility deregulation and restructuring and power technologies conference (DRPT'08), Nanjing, 6-9 April 2008, pp 764-770

[12] Esmail M, Amjady N, Shayanfar HA (2010) Stochastic congestion management in power markets using efficient scenario approaches. Energ Conver Manag 51(11):2285-2293

[13] Gan M, Xie KG, Li CY (2015) Transmission congestion evaluation of power systems using non-sequential Monte Carlo 
simulation technique. Int Trans Electr Energy Syst 25(4):636-647

[14] Billinton R, Huang DG (2008) Effects of load forecast uncertainty on bulk electric system reliability evaluation. IEEE Trans Power Syst 23(2):418-425

[15] Moghadasi SM, Kazemi A, Fotuhi-Firuzabad M et al (2008) Composite system reliability assessment incorporating an interline power-flow controller. IEEE Trans Power Deliv 23(2):1191-1199

[16] Guo L, Qiu QW, Liu J et al (2014) Power transmission risk assessment considering component. J Mod Power Syst Clean Energy 2(1):50-58. doi:10.1007/s40565-013-0042-y

[17] Jazaeri M, Farzinfar M, Razavi F (2014) Evaluation of the impacts of relay coordination on power system reliability. Int Trans Electr Energy Syst 25(12):3408-3421

[18] Bialek J (1996) Tracing the flow of electricity. IEE P-Gener Transm Distrib 143(4):313-320

[19] Strbac G, Kirschen D, Ahmed S (1998) Allocating transmission system usage on the basis of traceable contribution of generators and loads to flows. IEEE Trans Power Syst 13(2):527-534

[20] Narimani M, Hosseinian SH, Vahidi B (2014) A modified methodology in electricity tracing problems based on Bialek's method. Int J Electr Power Energy Syst 60:74-81

[21] Xie KG, Billinton R, Zhou JQ (2008) Tracing the unreliability contributions of power system components. Electr Power Compon Syst 36:1299-1309

[22] Xie KG, Billinton R (2009) Tracing the unreliability and recognizing the major unreliability contribution of network components. Reliab Eng Syst Safe 94(5):927-931

[23] Bialek JW, Kattuman PA (2004) Proportional sharing assumption in tracing methodology. IEE P-Gener Transm Distrib 151(4):526-532
[24] Xie KG, Hu B, Karki R (2011) Tracing the component unreliability contributions and recognizing the weak parts of a bulk power system. Euro Trans Electr Power 21(1):254-262

[25] Albrecht PF, Bhavaraju MP, Biggerstaff BE et al (1979) IEEE reliability test system: a report prepared by the reliability test system task force of the application of probability methods subcommittee. IEEE Trans Power Appar Syst 98(6):2047-2054

Ming GAN is a professor with the Department of Information Engineering, Logistical Engineering University, Chongqing, China. She received the Master degree in Electrical engineering from Chongqing University, Chongqing, China, in 1997. She is also a Ph.D. student with the School of Electrical Engineering, Chongqing University. Her research interests include power system steady analysis and logistics \& supply chain analysis.

Kaigui XIE is a professor with the School of Electrical Engineering, Chongqing University, China. He received the Ph.D. degree in Electrical engineering from Chongqing University of China in 2001. $\mathrm{He}$ is the author/coauthor of over 120 papers. His research interests include reliability, planning and analysis of power systems.

Chunyan LI is an associate professor with the School of Electrical Engineering, Chongqing University, China. She received the Ph.D. degree in Electrical engineering from Chongqing University of China in 2008. Her research interests include power system analysis and electricity market 